\title{
Estimates of certain paraxial diffraction integral operator and its generalized properties
}

\author{
Shrideh Al-Omari ${ }^{1 *}$ (D), Serkan Araci², Mohammed Al-Smadi ${ }^{3}$ Ghaleb Gumah and Hussam Alrabaiah ${ }^{4,5}$
}

\section{"Correspondence:}

s.k.q.alomari@fet.edu.jo

${ }^{1}$ Faculty of Engineering Technology,

Al-Balqa Applied University, 11134

Amman, Jordan

Full list of author information is

available at the end of the article

\section{Springer}

\begin{abstract}
This paper aims to discuss a generalization of certain paraxial diffraction integral operator in a class of generalized functions. At the start of this paper, we propose a convolution formula and establish certain convolution theorem. Then, with the addition to the convolution theorem, we consider a set of approximating identities and substantially employ our results in generating sets of integrable and locally integrable Boehmians. The said generalized integral operator is tested and declared to be one-to-one and onto mapping. Continuity of the generalized operator with respect to the convergence of the Boehmian spaces is obtained. Over and above, an inversion formula and consistency results are also counted.
\end{abstract}

MSC: 54C40; 14E20

Keywords: Optical Fresnel integral; Paraxial diffraction integral; Fractional Fourier integral; Boehmians

\section{Introduction}

The Fresnel integral operator is a paraxial diffraction integral operator, which describes the propagation of light from one transverse plane along the optical axis to another. In the one-dimensional case, it has been defined by aid of the integral equation (see, e.g., [1])

$$
\widehat{v}(x)=e^{i 2 \pi d / \lambda} e^{-i \pi / 4} \sqrt{\frac{1}{\lambda d}} \int_{-\infty}^{\infty} \exp \left(\frac{i \pi(x-\hat{x})^{2}}{\lambda d}\right) v(\hat{x}) d \dot{x}
$$

where $\widehat{v}$ is the output field related to the input field $v, d$ is the distance of the propagation of light, and $\lambda$ is the length of the wave. When $v_{a}$ specifies the fractional Fourier integral operator with order parameter $a$, the Fresnel integral operator can be explicitly decomposed into the fractional Fourier integral operator $v_{a}$ followed by magnification and chirp multiplication as follows (see, e.g., [2]):

$$
\widehat{v}(x)=e^{i 2 \pi d / \lambda} e^{-i a \pi / 4} \sqrt{\frac{1}{s M}} \exp \left(\frac{i \pi x^{2}}{\lambda \hat{R}}\right) v_{a}\left(\frac{x}{s M}\right),
$$

(c) The Author(s) 2020. This article is licensed under a Creative Commons Attribution 4.0 International License, which permits use, sharing, adaptation, distribution and reproduction in any medium or format, as long as you give appropriate credit to the original author(s) and the source, provide a link to the Creative Commons licence, and indicate if changes were made. The images or other third party material in this article are included in the article's Creative Commons licence, unless indicated otherwise in a credit line to the material. If material is not included in the article's Creative Commons licence and your intended use is not permitted by statutory regulation or exceeds the permitted use, you will need to obtain permission directly from the copyright holder. To view a copy of this licence, visit http://creativecommons.org/licenses/by/4.0/. 
where

$$
a=\frac{2}{\pi} \arctan \frac{\lambda d}{s^{2}}, \quad M=\sqrt{1+\frac{\lambda^{2} d^{2}}{s^{4}}}=\sec \frac{a \pi}{2} \quad \text { and } \quad \hat{R}=\frac{s^{4}+\lambda^{2} d^{2}}{\lambda^{2} d}=d \csc ^{2} \frac{a \pi}{2} .
$$

In the last era, the Fresnel integral operator has been implemented widely in Fourier optics (see, e.g., [2]), phase retrieval techniques (see, e.g., [3-5]), digital holography (see, e.g., [69] and [10-13]), biological imaging applications (see, e.g., [14, 15]), beam shaping (see, e.g., $[16,17])$, deformations (see, e.g., [18-20]), and many other areas of integral inequalities and the porous medium (see, e.g., [21-23]).

The name Boehmians is used to describe a space of objects that are defined as equivalence classes of pairs of sequences. Boehmians are constructed by using a set of axioms with two notions of convergence called $\delta$-convergence and $\Delta$-convergence. One of these axioms states that the denominator sequences are set to form a commutative semigroup with respect to a binary operation. To a context of Boehmians, certain variants of the Fresnel operator were extended to classes of Boehmians. In [24], a generalized Fresnel integral operator was discussed on a class of distributions and a space of integrable Boehmians. In [25], a diffraction Fresnel integral operator was discussed on strong Boehmian spaces. Likewise, in literature, various integral operators were discussed and thoroughly applied to various spaces of Boehmians, see for example [6, 10, 11, 26-37] and the references cited therein. However, in this approach, we discuss a new version of paraxial diffraction operators. We derive convolution products, convolution theorems, sets of Boehmians, and certain generalization of the diffraction operator. In contrast to profitable approaches furnished in [24] and [25], our results are new and distinguishable. In Sect. 2, we introduce convolution products and delta sequences, and extract various results. In Sect. 3, we provide several axioms and generate appropriate sets of Boehmians. In Sect. 4, we derive the generalized Fresnel integral and examine its desired properties.

\section{Convolution theorem and delta sequences}

In this section, we make use of the integral formula of the Fresnel operator and derive meritorious convolution products and delta sequences. On this basis, we consequently implement our results in constructing the integral convolution theorem. The very prerequisite convolution product to this variant can be earned as follows.

Definition 1 Let $v_{1}$ and $v_{2}$ be integrable functions in the space $L^{1}$. Then the product formula $*$ between $v_{1}$ and $v_{2}$ is defined as follows:

$$
v_{1} * v_{2}(\dot{x})=\int_{-\infty}^{\infty} v_{1}(w) v_{2}(\dot{x}-w) W(\dot{x}, w) d w
$$

where

$$
W(\dot{x}, w)=\exp \left(2 i w \frac{\pi(w-\hat{x})}{\lambda d}\right)
$$

is the proposed weight function, $d$ is the distance of the propagation of light, and $\lambda$ is the length of the wave. 
Denote by $L^{1}$ the set of all integrable functions on $\mathbb{R}$. Then, for the convenience of the reader, we drop the leading constant phase term $L=\exp (i 2 \pi d / \lambda) \exp (-i \pi / 4)$ for the rest of the analysis.

The convolution theorem of the classical Fresnel integral can be drawn as follows.

Theorem 2 Let $v_{1}$ and $v_{2}$ be two integrable functions in $L^{1}$. Then the Fresnel integral operator of the convolution product $v_{1} * v_{2}$ is given by

$$
\widehat{v_{1} * v_{2}}(x)=\frac{\exp \left(-i \pi x^{2} / \lambda d\right)}{L} \widehat{v}_{1}(x) \widehat{v}_{2}(x)
$$

where $L=\exp (i 2 \pi d / \lambda) \exp (-i \pi / 4)$.

Proof Let $v_{1}$ and $v_{2}$ be integrable functions over $\mathbb{R}$. Then, by applying (1), we routinely write

$$
\widehat{v_{1} * v_{2}}(x)=\int_{-\infty}^{\infty} \exp \left(\frac{i \pi(x-\dot{x})^{2}}{\lambda d}\right)\left(v_{1} * v_{2}\right)(x) d \dot{x}
$$

Hence, in view of (2), we get

$$
\widehat{v_{1} * v_{2}}(x)=\int_{-\infty}^{\infty} \exp \left(\frac{i \pi\left(x-x^{2}\right.}{\lambda d}\right) \int_{-\infty}^{\infty} v_{1}(w) v_{2}(\dot{x}-w) W(\dot{x}, w) d w d \dot{x}
$$

By taking into account the definition of $W$ and using the change of variables $z=x-w$ together with Fubini's theorem, we modify (3) as

$$
\widehat{v_{1} * v_{2}}(x)=\int_{-\infty}^{\infty} \widehat{v}_{1}(w) \int_{-\infty}^{\infty} \exp \left(\frac{i \pi\left(z^{2}+w^{2}-2(z+w) x+x^{2}\right)}{\lambda d}\right) \widehat{v}_{2}(z) d w d z
$$

Therefore, modifying and splitting out the exponent term under the integral sign in the previous equation yield

$$
\begin{aligned}
\widehat{v_{1} * v_{2}}(x) & =\int_{-\infty}^{\infty} v_{1}(w) \int_{-\infty}^{\infty} \exp \left(\frac{i \pi\left((z+w)^{2}+(w-x)^{2}-x^{2}\right)}{\lambda d}\right) v_{2}(z) d z d w \\
& =e^{-i \pi x^{2} / \lambda d} \int_{-\infty}^{\infty} \exp \left(\frac{i \pi(w-x)^{2}}{\lambda d}\right) v_{1}(w) d w \int_{-\infty}^{\infty} \exp \left(\frac{i \pi(z-x)^{2}}{\lambda d}\right) v_{2}(z) d z
\end{aligned}
$$

Hence, by taking into account the value of the leading constant phase term, we, indeed, obtain

$$
\widehat{v_{1} * v_{2}}(x)=\frac{\exp \left(-i \pi x^{2} / \lambda d\right)}{L} \widehat{v}_{1}(x) \widehat{v}_{2}(x)
$$

Hence, the proof of the theorem has been completed.

For deterministic needs, we establish the following very useful theorem.

Theorem 3 Let $v_{1}$ and $v_{2}$ be two integrable functions in $L^{1}$. Then the convolution product $v_{1} * v_{2}$ is an integrable function in $L^{1}$. 
Proof Let $v_{1}$ and $v_{2}$ be in $L^{1}$. Then, by aid of the fact that $|W(\dot{x}, w)| \leq 1$, we write

$$
\int_{-\infty}^{\infty}\left|v_{1} * v_{2}(\dot{x})\right| d \dot{x} \leq \int_{-\infty}^{\infty} \int_{-\infty}^{\infty}\left|v_{1}(w)\right|\left|v_{2}(\dot{x}-w)\right| d w d x
$$

Hence, by using Fubini's theorem, the previous integral inequality yields

$$
\int_{-\infty}^{\infty}\left|v_{1} * v_{2}(\dot{x})\right| d \dot{x} \leq \int_{-\infty}^{\infty}\left|v_{1}(w)\right| \int_{-\infty}^{\infty}\left|v_{2}(x-w)\right| d \dot{x} d w<\infty .
$$

The proof of the theorem is, therefore, completed.

The fact which deserves further attention is that $\widehat{v} \in L^{1}$ for every $v \in L^{1}$. Based upon this conjecture, we may think about the delta sequence choice. Indeed, by following (1), we deduce that

$$
\widehat{v}(0)=L \int_{-\infty}^{\infty} \exp \left(\frac{i \pi \dot{x}^{2}}{\lambda d}\right) \nu(x) d \dot{x} .
$$

Therefore, a wise choice of the description of delta sequences can be expressed in the following way.

Definition 4 Let $D$ denote the Schwartz space of test functions of compact supports over $\mathbb{R}$. By $\Delta$ we denote the collection of all sequences from $D$ such that

$$
\begin{aligned}
& \int_{-\infty}^{\infty} \exp \left(\frac{i \pi \dot{x}^{2}}{\lambda d}\right) w_{n}(\dot{x}) d x^{\prime}=1 . \\
& \int_{-\infty}^{\infty}\left|\exp \left(\frac{i \pi \dot{x}^{2}}{\lambda d}\right) w_{n}(\dot{x})\right| d \dot{x}<A, \quad A<\infty . \\
& \operatorname{supp}\left(w_{n}\right)(\hat{x}) \subseteq\left(-a_{n}, a_{n}\right), \quad a_{n} \rightarrow 0 \text { as } n \rightarrow \infty,
\end{aligned}
$$

where $\operatorname{supp}(w)$ is the support function of $w$. The following assertion holds for the delta sequences.

Theorem 5 Let $\left(w_{n}\right)$ and $\left(u_{n}\right)$ be two sequences in $\Delta$. Then $\left(w_{n} * u_{n}\right)$ is a sequence in $\Delta$.

Proof Firstly, we show that the integral equation

$$
\int_{-\infty}^{\infty} \exp \left(\frac{i \pi x^{2}}{\lambda d}\right)\left(w_{n} * u_{n}\right)(x) d x=1
$$

is satisfied for $\Delta$ sequences. By Theorem 2 we are allowed to write

$$
{\widehat{w_{n} * u_{n}}}(0)=\frac{1}{L} \widehat{w}_{n}(0) \widehat{u}_{n}(0) .
$$

Therefore, using (4), (7) may give

$$
\begin{aligned}
L \int_{-\infty}^{\infty} \exp \left(\frac{i \pi \dot{x}^{2}}{\lambda d}\right)\left(w_{n} * u_{n}\right)(\hat{x}) d \dot{x}= & \frac{1}{L}\left(L \int_{-\infty}^{\infty} \exp \left(\frac{i \pi \dot{x}^{2}}{\lambda d}\right) w_{n}(\dot{x}) d \dot{x}\right) \\
& \times\left(L \int_{-\infty}^{\infty} \exp \left(\frac{i \pi \dot{x}^{2}}{\lambda d}\right) u_{n}(\dot{x}) d x^{\prime}\right)
\end{aligned}
$$


Hence, owing to the fact that $\left(w_{n}\right)$ and $\left(u_{n}\right)$ are delta sequences in $\Delta$, (8) then reveals

$$
\int_{-\infty}^{\infty} \exp \left(\frac{i \pi \dot{x}^{2}}{\lambda d}\right)\left(w_{n} * u_{n}\right)(\dot{x}) d \dot{x}=1 \quad \text { for all } n \in \mathbb{N} \text {. }
$$

This proves (4). Proofs of (5) and (6), namely,

$$
\int_{-\infty}^{\infty}\left|\exp \left(\frac{i \pi \dot{x}^{2}}{\lambda d}\right)\left(w_{n} * u_{n}\right)(\hat{x})\right| d \dot{x}<A, \quad A<\infty
$$

and

$$
\operatorname{supp}\left(w_{n} * u_{n}\right)(\dot{x}) \subseteq\left(-a_{n}, a_{n}\right), \quad a_{n} \rightarrow 0 \text { as } n \rightarrow \infty,
$$

respectively, are straightforward results from the definitions. Hence, we delete the details of the similar proofs.

This completes the proof of the theorem.

\section{Fresnel integrable spaces of Boehmians}

We devote this section to the abstract construction of the space $B^{S}$ of integrable Boehmians and the space $H^{S}$ of ultra-Boehmians. We make a free use of the convolution theorem and define the convolution product which works in with the convolution $*$. To derive the generalized space $B^{S}$ of integrable Boehmians, we establish the following exemplary axioms.

Theorem 6 The following identities hold:

(i) $\left(v_{1}+v_{2}\right) * u_{n}=v_{1} * u_{n}+v_{2} * u_{n}$, where $v_{1}, v_{2} \in L^{1}, n \in \mathbb{N}$, and $u_{n} \in D$.

(ii) $(\alpha v) * u_{n}=\alpha\left(v * u_{n}\right)$, where $u_{n} \in D, v \in L^{1}, n \in \mathbb{N}$, and $\alpha \in \mathbb{C}$.

(iii) If $v_{n} \rightarrow v$ as $n \rightarrow \infty$ in $L^{1}$ and $u_{n} \in D$, then $v_{n} * \psi \rightarrow v * \psi$ as $n \rightarrow \infty$.

(iv) $v_{1} * v_{2}=v_{2} * v_{1}$ for $v_{1}, v_{2} \in L^{1}$.

(v) $v_{1} *\left(u_{1} * u_{2}\right)=\left(v_{1} * u_{1}\right) * u_{2}$, where $v_{1} \in L^{1}$ and $u_{1}, u_{2} \in D$.

Proof As the proofs of parts (i), (ii), and (iii) are straightforward consequences, following from simple integration, we prove part (iv) and part (v). By the convolution theorem, we get

$$
\widehat{v_{1} * v_{2}}=\frac{\exp \left(-i \pi x^{2} / \lambda d\right)}{L} \widehat{v}_{1} \widehat{v}_{2}=\frac{\exp \left(-i \pi x^{2} / \lambda d\right)}{L} \widehat{v}_{2} \widehat{v}_{1}=\widehat{v_{2} * v_{1}}
$$

Hence, by considering the inverse integral operator for both sides of the preceding equation, we complete the proof of (iv). The proof of part (v) is similar to the proof of part (iv). Therefore, we delete the details.

Hence, the proof of the theorem is completed.

Theorem 7 If $v \in L^{1}$ and $\left(w_{n}\right) \in \Delta$, then $v * w_{n} \rightarrow v$ as $n \rightarrow \infty$ in $L^{1}$.

Proof of this theorem follows from similar analysis given by the same author (see, e.g., $[24,25])$. 
The Boehmian space $B^{S}$ is based on the sets $L^{1}, D$, and $\Delta$, and the convolution product * is obtained. A Boehmian in $B^{S}$ may be introduced as

$$
x_{v_{n}}=\left(\frac{v_{n}}{w_{n}}\right)
$$

where $v_{n} \in L^{1}$ and $\left(w_{n}\right)$ is a delta sequence. If $x_{v_{n}}=\left(\frac{v_{n}}{w_{n}}\right)$ and $x_{u_{n}}=\left(\frac{u_{n}}{\varepsilon_{n}}\right)$ are in $B^{S}$, then it is possible to claim the space operations

$$
x_{v_{n}}+x_{u_{n}}=\left(\frac{v_{n} * w_{n}+u_{n} * w_{n}}{w_{n} * \varepsilon_{n}}\right) \quad \text { and } \beta x_{v_{n}}=\left(\frac{\beta v_{n}}{w_{n}}\right), \quad \forall \beta \in \mathbb{C} .
$$

For every $k \in \mathbb{N}$ and $\psi \in L^{1}$, we define on $B^{S}$ the following useful operations:

$$
x_{v_{n}} * x_{u_{n}}=\left(\frac{v_{n} * u_{n}}{w_{n} * \varepsilon_{n}}\right), \quad D^{k} x_{v_{n}}=\left(\frac{D^{k} v_{n}}{w_{n}}\right) \quad \text { and } \quad x_{v_{n}} * \psi=\left(\frac{v_{n} * \psi}{w_{n}}\right) \text {, }
$$

where $D^{k} x_{v_{n}}$ is the $k$ th derivative of $x_{v_{n}}$.

Definition 8 Let $x_{v_{n}, m}, x_{v_{n}} \in B^{S}, m=1,2,3, \ldots$ Then the sequence $\left(x_{v_{n}, m}\right)_{m=1}^{\infty}$ is $\delta$ convergent to $x_{v_{n}}$, denoted by $\delta-\lim _{m \rightarrow \infty} x_{v_{n}, m}=x_{v_{n}}$, provided that there can be found a delta sequence $\left(w_{n}\right)$ such that

(i) $\left(x_{v_{n}, m} * w_{k}\right),\left(x_{v_{n}} * w_{k}\right) \in L^{1}$ for all $m, k \in \mathbb{N}$, and

(ii) $\lim _{m \rightarrow \infty} x_{v_{n}, m} *_{\alpha, \beta} w_{k}=x_{v_{n}} * w_{k}$ in $L^{1}$ for every $k \in \mathbb{N}$.

Or, equivalently,

$\delta-\lim _{m \rightarrow \infty} x_{v_{n}, m}=x_{v_{n}}$ if and only if there are $u_{n, k}, u_{k} \in L^{1}$ and $\left(w_{k}\right) \in \Delta$ such that

(i) $w_{n}=\left(\frac{u_{n, k}}{w_{k}}\right), x_{v_{n}}=\left(\frac{u_{k}}{w_{k}}\right)$

(ii) to every $k \in N$, we have $\lim _{n \rightarrow \infty} u_{n, k}=u_{k}$ in $L^{1}$.

Definition 9 Let $x_{v_{n}, m}, x_{v_{n}} \in B^{S}, m=1,2,3, \ldots$ Then the sequence $\left(x_{v_{n}, m}\right)_{m=1}^{\infty}$ is $\Delta$ convergent to $x_{v_{n}}$, denoted by $\Delta-\lim _{m \rightarrow \infty} x_{v_{n}, m}=x_{v_{n}}$, provided that there can be found a delta sequence $\left(w_{n}\right)$ such that

(a) $\left(x_{v_{n}, m}-x_{v_{n}}\right) * w_{n} \in L^{1}(\forall m \in \mathbb{N})$;

(b) $\lim _{m \rightarrow \infty}\left(x_{v_{n}, m}-x_{v_{n}}\right) * w_{n}=0$ in $L^{1}$.

Theorem 10 Let $\left(w_{n}\right) \in \Delta$ be fixed and $u \in L^{1}$. Then the mapping

$$
u \rightarrow x_{v_{n}},
$$

$x_{v_{n}}=\left(\frac{u * w_{n}}{w_{n}}\right)$, is a one-one map from the space $L^{1}$ into the Boehmian space $B^{S}$.

From (9), it can be read that $L^{1}$ has an identification with a subspace in $B^{S}$.

Theorem 11 Let $\left(w_{n}\right) \in \Delta$.If $u_{n} \rightarrow u$ in $L^{1}$ as $n \rightarrow \infty$, then, for all $k \in N, u_{n} * u_{k} \rightarrow u * u_{k}$ as $n \rightarrow \infty$.

Theorem 11 clearly shows that

$$
x_{v_{n}, m} \rightarrow x_{v_{n}} \text { in } B^{S} \text { as } m \rightarrow \infty .
$$

Moreover, the above theorem indeed leads to the following statement. 
Theorem 12 The embedding of the space $L^{1}$ into the Boehmian space $B^{S}$ defined by (9) is continuous.

Now, let us evaluate the ultra-Boehmian space $H^{S}$. Introduce the set $I^{S}$ to be the set of all Fresnel operators of all $L^{1}$ elements. Similarly, denote by $D^{S}\left(\right.$ or $\left.\Delta^{S}\right)$ the set of all Fresnel transforms of all $D$ ( or $\Delta$ ) elements, respectively. The product formula $\triangleright$ is defined for $H^{S}$ as follows.

Definition 13 Let $\widehat{v} \in I^{S}$ and $u^{S} \in D^{S}$. Then we define

$$
\widehat{v} \triangleright \widehat{u}=\frac{\exp \left(-i \pi x^{2} / \lambda d\right)}{L} \widehat{v} \widehat{u} .
$$

We prove the following necessary axioms.

Theorem 14 The following hold:

(i) $\left(\widehat{v}_{1}+\widehat{v}_{2}\right) \triangleright \widehat{u}=\widehat{v}_{1} \triangleright \widehat{u}+\widehat{v}_{2} \triangleright \widehat{u}$ for every $\widehat{v}_{1}, \widehat{v}_{2} \in I^{S}$ and $\widehat{u} \in D^{S}$.

(ii) $(\beta \widehat{v}) \triangleright \widehat{u}=\beta(\widehat{v} \triangleright \widehat{u})$ for every $\widehat{v}_{2} \in I^{S}, \widehat{u} \in D^{S}$, and $\beta \in \mathbb{C}$; the space of complex numbers.

(iii) If $\widehat{v}_{n} \rightarrow \widehat{v}$ as $n \rightarrow \infty$ and $\widehat{u} \in D^{S}$, then $\widehat{v}_{n} \triangleright \widehat{u} \rightarrow \widehat{v} \triangleright \widehat{u}$ as $n \rightarrow \infty$ in $I^{S}$.

(iv) $\widehat{v}_{1} \triangleright \widehat{v}_{2}=\widehat{v}_{2} \triangleright \widehat{v}_{1}$ for $\widehat{v}_{1}, \widehat{v}_{2} \in I^{S}$.

(v) If $\widehat{v} \in I^{S}$ and $\left(\widehat{w}_{n}\right) \in \Delta^{S}$, then $\widehat{v} \triangleright \widehat{w}_{n} \rightarrow \widehat{v}$ in $I^{S}$ as $n \rightarrow \infty$.

Proof Proof of (i). Let $\widehat{v}_{2} \in I^{S}$ and $\widehat{u} \in D^{S}$ be the Fresnel operator of the elements $u \in L^{1}$ and $v \in D$, respectively. Then, by (10), we have

$$
\begin{aligned}
\left(\widehat{v}_{1}+\widehat{v}_{2}\right) \triangleright \widehat{u} & =\frac{\exp \left(-i \pi x^{2} / \lambda d\right)}{L}\left(\widehat{v}_{1}+\widehat{v}_{2}\right) \widehat{u} \\
& =\frac{\exp \left(-i \pi x^{2} / \lambda d\right)}{L} \widehat{v}_{1} \widehat{u}+\frac{\exp \left(-i \pi x^{2} / \lambda d\right)}{L} \widehat{v}_{2} \widehat{u} \\
& =\widehat{v}_{1} \triangleright \widehat{u}+\widehat{v}_{2} \triangleright \widehat{u} .
\end{aligned}
$$

Proof of (ii) follows from a similar argument used in the proof of (i). To prove (iii), let $\widehat{u} \in D^{S}$ be the Fresnel operator of $u \in D$ and $\widehat{v}_{n}, \widehat{v}$ be the Fresnel operators of $v_{n}$ and $v \in L^{1}$, respectively. Then, by (10) and Theorem 2 , we proceed to write

$$
\begin{aligned}
\left(\widehat{v}_{n} \triangleright \widehat{u}-\widehat{v} \triangleright \widehat{u}\right) & =\frac{\exp \left(-i \pi x^{2} / \lambda d\right)}{L} \widehat{v}_{n} \widehat{u}-\frac{\exp \left(-i \pi x^{2} / \lambda d\right)}{L} \widehat{v} \widehat{u} \\
& =\widehat{v_{n} * u}-\widehat{v * u} \\
& =v_{n} * \widehat{u-v} * u \\
& =\left(v_{n}-\widehat{v)} * u\right. \\
& =\frac{\exp \left(-i \pi x^{2} / \lambda d\right) \widehat{v_{n}-v \widehat{u}}}{L} .
\end{aligned}
$$

Hence, by the assumption that $\widehat{v}_{n} \rightarrow \widehat{v}$ as $n \rightarrow \infty$, the right-hand side of the above equation approaches zero as $n \rightarrow \infty$. Hence, we have obtained $\widehat{v}_{n} \triangleright \widehat{u} \rightarrow \widehat{v} \triangleright \widehat{u}$ as $n \rightarrow \infty$. The proofs of (iv) and (v) are similar.

This completes the proof of the theorem. 
The Boehmian space $H^{S}$ is approved by the above theorem. Every member of $H^{S}$ is defined as

$$
x_{\widehat{v}_{n}}=\left(\frac{\widehat{v}_{n}}{\widehat{w}_{n}}\right) .
$$

If $x_{\widehat{v}_{n}}=\left(\frac{\widehat{v}_{n}}{\widehat{w}_{n}}\right)$ and $x_{\widehat{u}_{n}}=\left(\frac{\widehat{u}_{n}}{\widehat{\varepsilon}_{n}}\right)$ are in $H^{S}$, then we define

$$
x_{\widehat{v}_{n}}+x_{\widehat{u}_{n}}=\frac{\widehat{v} \triangleright \widehat{w}_{n}+\widehat{u}_{n} \triangleright \widehat{w}_{n}}{\widehat{w}_{n} \triangleright \widehat{\varepsilon}_{n}} \quad \text { and } \quad \beta x_{\widehat{v}_{n}}=\left(\frac{\beta \widehat{v}}{\widehat{w}_{n}}\right), \quad \forall \beta \in \mathbb{C} .
$$

Also, for $k \in \mathbb{R}$ and $\hat{\psi} \in I^{S}$, we define operations on $H^{S}$ as

$$
x_{\widehat{v}_{n}} \triangleright x_{\widehat{u}_{n}}=\left(\frac{\widehat{v} \triangleright \widehat{u}_{n}}{\widehat{w}_{n} \triangleright \widehat{\varepsilon}_{n}}\right), \quad D^{k} x_{\widehat{v}_{n}}=\left(\frac{D^{k} \widehat{v}}{\widehat{w}_{n}}\right) \quad \text { and } \quad x_{\widehat{v}_{n}} \triangleright \hat{\psi}=\left(\frac{\widehat{v} \triangleright \hat{\psi}}{\widehat{w}_{n}}\right)
$$

where $D^{k} x_{\widehat{v}_{n}}$ is the $k$ th derivative of $x_{\widehat{v}_{n}}$.

Definition 15 Let $x_{\widehat{v}_{n}, m}, x_{\widehat{v}_{n}} \in H^{S}$ for $m=1,2,3, \ldots$ Then the convergence in $H^{S}$ is defined as follows.

(i) The sequence $\left(x_{\widehat{v}_{n}, m}\right)_{m=1}^{\infty}$ is said to be $\delta$-convergent to $x_{\widehat{v}_{n}}$, denoted by $\delta-\lim _{m \rightarrow \infty} x_{\widehat{v}_{n}, m}=x_{\widehat{v}_{n}}$, provided that there can be found a delta sequence $\left(\widehat{w}_{n}\right)$ such that

(a) $\left(x_{\widehat{v}_{n}, m} \triangleright w_{k}\right),\left(x_{\widehat{v}_{n}} \triangleright \widehat{w}_{k}\right) \in I^{S}$ for all $n, k \in \mathbb{N}$.

(b) $\lim _{m \rightarrow \infty} x_{\widehat{x}_{n}, m} \triangleright \widehat{w}_{k}=x_{\widehat{v}_{n}} \triangleright \widehat{w}_{k}$ in $I^{S}$ for every $k \in \mathbb{N}$.

(ii) The sequence $\left(x_{\widehat{v}_{n}, m}\right)_{m=1}^{\infty}$ is $\Delta^{S}$-convergent to $x_{\widehat{v}_{n}}$, denoted by $\Delta^{S}-\lim _{m \rightarrow \infty} x_{\widehat{v}_{n}, m}=x_{\widehat{v}_{n}}$, provided that there can be found a delta sequence $(\widehat{w})$ such that

(a) $\left(x_{\widehat{v}_{n}, m}-x_{\widehat{v}_{n}}\right) \triangleright \widehat{w}_{n} \in I^{S}(\forall m \in \mathbb{N})$;

(b) $\lim _{m \rightarrow \infty}\left(x_{\widehat{v}_{n}, m}-x_{\widehat{v}_{n}}\right) \triangleright \widehat{w}_{n}=0$ in $I^{S}$.

Theorem 16 Let $(\widehat{w}) \in \Delta^{S}$ be fixed and $u \in I^{S}$. Then the mapping defined by

$$
u \rightarrow x_{\widehat{v}_{n}} \text {, }
$$

where $x_{\widehat{v}_{n}}=\left(\frac{u \triangleright \widehat{w}}{\widehat{w}}\right)$, is a one-to-one mapping $I^{S} \rightarrow H^{S}$.

From (11), it can be read that $I^{S}$ can be identified as subspaces of $H^{S}$.

Theorem 17 Let $(\widehat{w}) \in \Delta^{S}$. Then if $u_{n} \rightarrow u$ in $I^{S}$ as $n \rightarrow \infty$, then, for all $k \in \mathbb{N}, u_{n} \triangleright u_{k} \rightarrow$ $u \triangleright u_{k}$ as $n \rightarrow \infty$. That is, $x_{\widehat{v}_{n}, m} \rightarrow x_{\widehat{v}_{n}}$ in $H^{S}$ as $m \rightarrow \infty$.

The above theorem leads to the following:

Theorem 18 The mapping defined by (9) is a continuous embedding of the space $I^{S}$ into the ultra-Boehmian space $H^{S}$. 


\section{The boehmian Fresnel operators}

By the former analysis, we define the Fresnel operator of the Boehmian $x_{v_{n}}=\left(\frac{v_{n}}{w_{n}}\right)$ in $B^{S}$ as a Boehmian in $H^{S}$ defined by

$$
\widehat{x_{v_{n}}}=x_{\widehat{v_{n}}},
$$

where $x_{\widehat{v}_{n}}=\left(\frac{\widehat{v}_{n}}{\widehat{w}_{n}}\right)$.

Theorem 19 Let $x_{v_{n}}=\left(\frac{v_{n}}{w_{n}}\right)$. Then the mapping $x_{v_{n}} \rightarrow x_{\widehat{v}_{n}}$ from $B^{S} \rightarrow H^{S}$ defined by $\widehat{x_{v_{n}}}=$ $x_{\widehat{v}_{n}}$ coincides with the classical Fresnel operator $L^{1} \rightarrow I^{S}$.

Proof Let $w \in L^{1}$. Then $w$ can be identified in $B^{S}$ as $x_{v_{n}}$, where $x_{v_{n}}=\left(\frac{w * v_{n}}{v_{n}}\right)$, which is the representation of $w$ in $B^{S}$. Now, by employing Theorem 2, we obtain

$$
x_{\widehat{v}_{n}}=\left(\frac{\widehat{w * v_{n}}}{\widehat{v}_{n}}\right)=\frac{\exp \left(-i \pi x^{2} / \lambda d\right)}{L}\left(\frac{\hat{w} \widehat{v}_{n}}{\widehat{v}_{n}}\right)=\left(\frac{\hat{w} \triangleright \widehat{v}_{n}}{\widehat{v}_{n}}\right)
$$

which is the representation of $\hat{w}$ in $H^{S}$.

The proof is, therefore, finished.

Theorem 20 Let $x_{v_{n}}=\left(\frac{v_{n}}{w_{n}}\right)$ and $\widehat{x_{v_{n}}}=x_{\widehat{v}_{n}}$. Then the mapping $x_{v_{n}} \rightarrow x_{\widehat{v}_{n}}$ from $B^{S}$ onto $H^{S}$, defined by $\widehat{x_{v_{n}}}=x_{\widehat{v}_{n}}$, is linear and continuous with respect to the convergence of the Boehmian spaces.

A similar proof for this theorem is available in various citations (see, e.g., [28] and [29]). Hence it is omitted.

We introduce the inverse operator of the operator $x_{\widehat{v}_{n}}$ as follows.

Definition 21 Let $x_{\widehat{v}_{n}} \in H^{S}, x_{\widehat{v}_{n}}=\left(\frac{\widehat{v}_{n}}{\widehat{w}_{n}}\right)$ for $x_{v_{n}}=\left(\frac{v_{n}}{w_{n}}\right)$. We define the inverse operator of $x_{\widehat{v}_{n}}$ as

$$
\widehat{v}^{-1} x_{\widehat{v}_{n}}=x_{v_{n}} .
$$

We derive the following inversion properties.

Theorem 22 Let $x_{\widehat{v}_{n}} \in H^{S}$ and $w=\widehat{v}$ for $v \in L^{1}$. The inverse operator $x_{\widehat{v}_{n}} \rightarrow x_{v_{n}}$ is linear. Moreover, it satisfies

$$
\widehat{v}^{-1}\left(x_{\widehat{v}_{n}} \triangleright w\right)=x_{v_{n}} * v \text { and } \widehat{x_{v_{n}} * v}=x_{\widehat{v}_{n}} \triangleright w .
$$

Proof Consider two arbitrary Boehmians $x_{\widehat{v}_{n}}$ and $x_{\widehat{u}_{n}}$ in $H^{S}$ defined, respectively, by $x_{\widehat{v}_{n}}=$ $\left(\frac{\widehat{v}_{n}}{\widehat{w}_{n}}\right)$ and $x_{\widehat{u}_{n}}=\left(\frac{\widehat{u}_{n}}{\widehat{\epsilon}_{n}}\right)$. Then, for all $n \in \mathbb{N}$, Theorem 2 and the linearity of the integral reveal

$$
x_{\widehat{v}_{n}}+x_{\widehat{u}_{n}}=\left(\frac{\widehat{v}_{n} \triangleright \widehat{\epsilon}_{n}+\widehat{u}_{n} \triangleright \widehat{w}_{n}}{\widehat{w}_{n} \triangleright \widehat{\epsilon}_{n}}\right)=\left(\frac{\frac{\exp \left(-i \pi x^{2} / \lambda d\right)}{L}\left(\widehat{v_{n} * \epsilon_{n}}+\widehat{u_{n} * w_{n}}\right)}{\frac{\exp \left(-i \pi x^{2} / \lambda d\right)}{L}\left(\widehat{w_{n} * \epsilon_{n}}\right)}\right) .
$$


Hence, Definition 21 and the notion of addition in $B^{S}$ imply

$$
\widehat{v}^{-1}\left(x_{\widehat{v}_{n}}+x_{\widehat{u}_{n}}\right)=\left(\frac{v_{n} * \epsilon_{n}+u_{n} * w_{n}}{w_{n} * \epsilon_{n}}\right)=\left(\frac{v_{n}}{w_{n}}\right)+\left(\frac{u_{n}}{\epsilon_{n}}\right) .
$$

In notations, this gives

$$
\widehat{v}^{-1}\left(x_{\widehat{v}_{n}}+x_{\widehat{u}_{n}}\right)=x_{v_{n}}+x_{u_{n}}
$$

To complete the proof of linearity, indeed, for some $\eta \in \mathbb{C}$ and all $n \in \mathbb{N}$, we have

$$
\widehat{v}^{-1}\left(\eta x_{\widehat{v}_{n}}\right)=\eta \widehat{v}^{-1} x_{\widehat{v}_{n}} .
$$

This proves the linearity condition. To show $\widehat{v}^{-1}\left(x_{\widehat{v}_{n}} \triangleright w\right)=x_{v_{n}} * v$, we assume $x_{v_{n}}=\left(\frac{v_{n}}{w_{n}}\right)$ in $H^{S}$. Then, for every $w \in L^{1}$, we have $\widehat{v}^{-1}\left(x_{\widehat{v}_{n}} \triangleright w\right)=\left(\frac{\widehat{v}_{n} \triangleright \widehat{v}}{\widehat{w}_{n}}\right)$. By using Theorem 2 and Definition 21, we get

$$
\widehat{v}^{-1}\left(x_{\widehat{v}_{n}} \triangleright w\right)=\widehat{v}^{-1}\left(\frac{\widehat{v_{n} * v}}{\widehat{w}_{n}}\right)=\left(\frac{v_{n}}{w_{n}} * v\right)=x_{v_{n}} * v .
$$

Proof of the part $\widehat{x_{v_{n}} * v}=x_{\widehat{v}_{n}} \triangleright w$ is almost similar to the inversion case.

This finishes the proof of the theorem.

\section{Conclusion}

In this article, the paraxial diffraction integral operator has been extended to a class of Boehmians, and the new operator has been obtained as a Boehmian. Many properties of the generalized diffraction integral operator coincide with the estimated properties of the classical operator. Various embeddings are also defined.

\section{Acknowledgements}

The authors would like to thank their friends for their support.

\section{Funding}

No funding sources to be declared.

Availability of data and materials

Please contact the author for data requests.

\section{Competing interests}

The authors declare that they have no competing interests regarding the publication of the article.

Authors' contributions

The authors contributed equally and significantly in writing this paper. All authors read and approved the final manuscript.

\section{Author details}

${ }^{1}$ Faculty of Engineering Technology, Al-Balqa Applied University, 11134 Amman, Jordan. ${ }^{2}$ Department of Economics, Faculty of Economics, Administrative and Social Sciences, Hasan Kalyoncu University, TR-27410 Gaziantep, Turkey. ${ }^{3}$ Department of Applied Science, Ajloun College, Al-Balqa Applied University, Ajloun 26816, Jordan. ${ }^{4}$ College of Engineering, Al Ain University, Al Ain, UAE. ${ }^{5}$ Department of Mathematics, Tafila Technical University, Tafila, Jordan.

\section{Publisher's Note}

Springer Nature remains neutral with regard to jurisdictional claims in published maps and institutional affiliations. 


\section{References}

1. Ozaktas, H.M., Arık, S.O., Coşkun, T.: Fundamental structure of Fresnel diffraction: natural sampling grid and the fractional Fourier transform. Opt. Lett. 36(13), 2524-2526 (2011)

2. Ozaktas, H.M., Zalevsky, Z., Kutay, M.A.: The Fractional Fourier Transform with Applications in Optics and Signal Processing. Wiley, New York (2001)

3. Kelly, D.P.: Numerical calculation of the Fresnel transform. J. Opt. Soc. Am. 31(4), 755-764 (2014)

4. Fienup, J.R.: Phase retrieval algorithms: a comparison. Appl. Opt. 21, 2758-2769 (1982)

5. Almoro, P.F., Gundu, P.N., Hanson, S.G.: Numerical correction of aberrations via phase retrieval with speckle illumination. Opt. Lett. 34, 521-523 (2009)

6. Loonker, D., Banerji, P.K., Kalla, S.L.: Wavelet transform of fractional integrals for integrable Boehmians. Appl. Appl. Math. 5(1), 1-10 (2010)

7. Kelly, D.P., Hennelly, B.M., Pandey, N., Naughton, T.J., Rhodes, W.T.: Resolution limits in practical digital holographic systems. Opt. Eng. 48, 095801 (2009)

8. Picart, P., Leval, J., Mounier, D., Gougeon, S.: Some opportunities for vibration analysis with time averaging in digital Fresnel holography. Appl. Opt. 44, 337-343 (2005)

9. Pedrini, G., Frnning, P., Tiziani, H.J., Gusev, M.E.: Pulsed digital holography for high-speed contouring that uses a two-wavelength method. Appl. Opt. 38, 3460-3467 (1999)

10. Boehme, T.K.: The support of Mikusiński operators. Trans. Am. Math. Soc. 176, 319-334 (1973)

11. Ganesan, C.: Weighted ultra distributions and Boehmians. Int. J. Math. Anal. 4, 703-712 (2010)

12. Molony, K.M., Hennelly, B.M., Kelly, D.P., Naughton, T.J.: Reconstruction algorithms applied to in-line Gabor digital holographic microscopy. Opt. Commun. 283, 903-909 (2010)

13. Monaghan, D.S., Kelly, D.P., Pandey, N., Hennelly, B.M.: Twin removal in digital holography using diffuse illumination. Opt. Lett. 34, 3610-3612 (2009)

14. Meinecke, T., Sabitov, N., Sinzinger, S.: Information extraction from digital holograms for particle flow analysis. Appl. Opt. 49, 2446-2455 (2010)

15. Teague, M.R.: Deterministic phase retrieval: a Green's function solution. J. Opt. Soc. Am. 73, 1434-1441 (1983)

16. Arrizin, V., Testorf, M., Sinzinger, S., Jahns, J.: Iterative optimization of phase-only diffractive optical elements based on a lenslet array. J. Opt. Soc. Am. A 17, 2157-2164 (2000)

17. Sheppard, C.J.R.: Three-dimensional phase imaging with the intensity transport equation. Appl. Opt. 41, 5951-5955 (2002)

18. Kleindienst, R., Moeller, L., Sinzinger, S.: Highly efficient refractive Gaussian-to-tophat beam shaper for compact terahertz imager. Appl. Opt. 49, 1757-1763 (2010)

19. Eriksson, I., Haglund, P., Powell, J., Sjodahl, M., Kaplan, A.F.H.: Holographic measurement of thermal distortion during laser spot welding. Opt. Eng. 51, 030501 (2012)

20. Angelsky, O.V., Maksimyak, A.P., Maksimyak, P.P., Hanson, S.G.: Optical correlation diagnostics of rough surfaces with large surface inhomogeneities. Opt. Express 14, 7299-7311 (2006)

21. Agarwal, R., Yadav, M.P., Baleanu, D., Purohit, S.D.: Existence and uniqueness of miscible flow equation through porous media with a nonsingular fractional derivative. AIMS Math. 5(2), 1062-1073 (2020)

22. Mishra, A.M., Baleanu, D., Tchier, F., Purohit, S.D.: Certain results comprising the weighted Chebyshev function using pathway fractional integrals. Mathematics 7, 896 (2019)

23. Li, T., Pintus, N., Viglialoro, G.: Properties of solutions to porous medium problems with different sources and boundary conditions. Z. Angew. Math. Phys. 70, 86 (2019)

24. Goodman, J.W.: Speckle Phenomena in Optics. Roberts and Company (2007)

25. Al-Omari, S.K.Q., Baleanu, D.: Quaternion Fourier integral operators for spaces of generalized quaternions. Math. Methods Appl. Sci. 41, 9477-9484 (2018)

26. Al-Omari, S.K., Baleanu, D.: A quadratic-phase integral operator for sets of generalized integrable functions. Math. Methods Appl. Sci. 43, 1-9 (2020)

27. Al-Omari, S.K.Q., Baleanu, D.: Some remarks on short-time Fourier integral operators and classes of rapidly decaying functions. Math. Methods Appl. Sci. 42(16), 5354-5361 (2019)

28. Al-Omari, S.K.Q.: Some characteristics of $S$ transforms in a class of rapidly decreasing Boehmians. J. Pseudo-Differ. Oper. Appl. 5(4), 527-537 (2014)

29. Al-Omari, S.K.Q., Kilicman, A.: An estimate of Sumudu transform for Boehmians. Adv. Differ. Equ. 2013, 77 (2013)

30. Roopkumar, R.: An extension of distributional wavelet transform. Colloq. Math. 115(2), 195-206 (2009)

31. Mikusiński, P.: Convergence of Boehmians. Jpn. J. Math. New Ser. 9(1), 159-179 (1983)

32. Al-Omari, S.K.Q.: Some estimate of a generalized Bessel-Struve transform on certain space of generalized functions. Ukr. Math. J. 69(9), 1155-1165 (2017)

33. Al-Omari, S.K.Q.: Some estimate for the extended Fresnel transform and its properties in a class of Boehmians. J. Appl. Funct. Anal. 10(1/2), 266-280 (2015)

34. Al-Omari, S.K.Q.: A study on a class of modified Bessel-type integrals in a Fréchet space of Boehmians. Bol. Soc. Parana Mat. 38(4), 145-156 (2020)

35. Al-Omari, S.K.Q., Kilicman, A.: On generalized Hartley-Hilbert and Fourier-Hilbert transforms. Adv. Differ. Equ. 2012, $232(2012)$

36. Al-Omari, S.K.Q.: Natural transform in Boehmian spaces. Nonlinear Stud. 22(2), 291-297 (2015)

37. Bhuvaneswari, R., Karunakaran, V.: Boehmians of type $S$ and their Fourier transforms. Ann. Univ. Mariae Curie-Skłodowska, Sect. A 64(1), 27-43 (2010) 